\title{
A Non-dimensional Formulation of the Passive Bidomain Equation
}

\author{
Peter R. Johnston \\ School of Biomolecular and Physical Sciences, Griffith University, Nathan, QLD, \\ Australia, 4111
}

\begin{abstract}
Simulation studies of ST depression arising from subendocardial ischemia show a marked difference in the resulting epicardial potential distributions depending on which of the three common experimentally determined bidomain conductivity data sets is chosen. Here, the governing equation is rendered non-dimensional by dividing by the difference in normal and ischemic transmembrane potentials during the ST segment and by the sum of the intra and extracellular conductivities in the transverse direction, yielding the ratio of the sum of the intra and extracellular longitudinal conductivities divided by the sum of the intra and extracellular transverse conductivities as a dimensionless group. Averaging this ratio over the three sets of experimentally determined data gives the value of $3.21 \pm 0.08$. The effect of this narrow range means that the left hand side of the governing equation can be considered, as a good approximation, to be the same for all these sets of conductivity data. Hence, the right hand of the non-dimensional differential equation contains all the necessary information to compare the effect different conductivity data sets have on the epicardial potential distribution. As an example, an explanation is given as to why values from one data set gives rise to epicardial distributions which are markedly different from those obtained from the other two data sets.
\end{abstract}


Keywords: Bidomain equation, electrocardiography, Mathematical Model

\section{Introduction}

Consider the passive bidomain equation [1]

$$
\nabla \cdot\left(\mathbf{M}_{e}+\mathbf{M}_{i}\right) \nabla \phi_{e}=-\nabla \cdot \mathbf{M}_{i} \nabla \phi_{m}
$$

for predicting the extracellular electric potential, $\phi_{e}$, in cardiac tissue for a given transmembrane potential distribution, $\phi_{m}$. The conductivity tensors $\mathbf{M}_{i}$ and $\mathbf{M}_{e}$ contain information about the local fiber direction and the intracellular $(i)$ and extracellular $(e)$ conductivity in the directions along and across the fibers.

Assuming that the tissue is in contact with a blood mass, the potential in the blood,$\phi_{b}$, is governed by Laplace's equation

$$
\nabla^{2} \phi_{b}=0
$$

with continuity of potential and current across the interface.

This form of the bidomain equation has been used previously to study epicardial potential distributions from subendocardial ischemia $[1,2,3,4$, $5,6]$, with particular emphasis on ST segment changes. Most studies use experimentally derived conductivity values (see Table 1) [7, 8, 9]. Another popular idea is to use artificially created conductivity values constructed in accordance with the ideas suggested by Roth [10]. Roth's idea was to introduce the ratio $\epsilon$ given by

$$
\epsilon=1-\frac{g_{e l} / g_{e t}}{g_{i l} / g_{i t}}
$$

where $\epsilon$ is fixed at 0.75 . This value of $\epsilon$ is based on averaging the specific quantities over the three experimentally derived data sets. These three values 
of $\epsilon$ are given in Table 1 and have an average value of 0.761 and standard deviation of 0.072 .

A disturbing feature of the variation in the experimentally obtained conductivity values is the difference in epicardial potential distributions observed for geometrically simple models of cardiac tissue, as presented in previous studies of models of ST segment shift due to partial thickness ischemia $[3,4,5]$. These studies show that the data of Clerc [7] and Roberts et al. [8] give similar potential distributions in that there are three negative potential wells and two peaks of positive potential as well as large potential gradients over two sides of the ischemic region. However, the data of Roberts and Scher [9] produce only negative potential, but still show the large potential gradient above the ischemic boundary. These observations apply to slab [3, 4], cylindrical [5] and ellipsoidal models of the left ventricle.

The object of this paper is to offer a possible explanation for the differences in observed epicardial potential distributions obtained from the different sets of experimentally determined conductivity values. This objective is achieved by proposing a new non-dimensional formulation of the passive bidomain equation so as to render the left hand side of the equation "almost" independent of the conductivity values. The result of this non-dimensional formulation focuses the effect of the conductivity changes in the right hand side of equation (1), which can be evaluated numerically, or in simple cases, analytically, and visualized in three dimensional space.

In the next section the structure of the conductivity tensor is considered and a new non-dimensional formulation for the passive bidomain equation introduced. Section 3 shows a simple example with the new formulation and in Section 4 a possible explanation for the difference in potential distributions is presented. Finally, as part of the conclusion, a new nominal set of 
conductivity parameters is proposed.

\section{Methods}

\subsection{The conductivity Tensor}

Before considering the passive bidomain equation (1), consider in detail the conductivity tensors $\mathbf{M}_{i}$ and $\mathbf{M}_{e}$. Generally, these tensors take the form $[11]$

$$
\mathbf{M}_{h}=\mathbf{A G}_{h} \mathbf{A}^{T}
$$

for $h=i$ or $e$, where $\mathbf{G}_{h}$ is a diagonal matrix containing the conductivity values along the fiber direction, $g_{h l}$, across the sheets of fibers, $g_{h t}$, and between the sheets of fibers, $g_{h n}$. Here it will be assumed that $g_{h t}=g_{h n}$. The matrix $\mathbf{A}=\left\{a_{i j}\right\}$ is a rotation matrix mapping the local fiber direction into the global coordinate system and is, by definition, orthonormal.

Writing the matrix product in full gives

$$
\mathbf{M}_{h}=\mathbf{A G}_{h} \mathbf{A}^{T}=\left(\begin{array}{ccc}
a_{11} & a_{12} & a_{13} \\
a_{21} & a_{22} & a_{23} \\
a_{31} & a_{32} & a_{33}
\end{array}\right)\left(\begin{array}{ccc}
g_{h l} & 0 & 0 \\
0 & g_{h t} & 0 \\
0 & 0 & g_{h t}
\end{array}\right)\left(\begin{array}{lll}
a_{11} & a_{21} & a_{31} \\
a_{12} & a_{22} & a_{32} \\
a_{13} & a_{23} & a_{33}
\end{array}\right)
$$

Following some manipulation and using the fact that $\mathbf{A}$ is orthonormal it can be shown that

$$
\mathbf{M}_{h}=\left(\begin{array}{ccc}
\left(g_{h l}-g_{h t}\right) a_{11}^{2}+g_{h t} & \left(g_{h l}-g_{h t}\right) a_{11} a_{21} & \left(g_{h l}-g_{h t}\right) a_{11} a_{31} \\
\left(g_{h l}-g_{h t}\right) a_{11} a_{21} & \left(g_{h l}-g_{h t}\right) a_{21}^{2}+g_{h t} & \left(g_{h l}-g_{h t}\right) a_{21} a_{31} \\
\left(g_{h l}-g_{h t}\right) a_{11} a_{31} & \left(g_{h l}-g_{h t}\right) a_{21} a_{31} & \left(g_{h l}-g_{h t}\right) a_{31}^{2}+g_{h t}
\end{array}\right)
$$

which is of a similar form to that presented elsewhere $[12,13]$. It now follows that the diagonal elements of the tensor $\mathbf{M}_{i}+\mathbf{M}_{e}$ take the form

$$
\left(\mathbf{M}_{i}+\mathbf{M}_{e}\right)_{k k}=\left(g_{i l}-g_{i t}+g_{e l}-g_{e t}\right) a_{k 1}^{2}+\left(g_{i t}+g_{e t}\right)
$$


and the off-diagonal elements have the form

$$
\left(\mathbf{M}_{i}+\mathbf{M}_{e}\right)_{k j}=\left(g_{i l}-g_{i t}+g_{e l}-g_{e t}\right) a_{k 1} a_{j 1}
$$

Next, if the tensor $\mathbf{M}_{i}+\mathbf{M}_{e}$ is divided by the quantity $g_{i t}+g_{e t}$, the diagonal elements become

$$
\frac{\left(\mathbf{M}_{i}+\mathbf{M}_{e}\right)_{k k}}{g_{i t}+g_{e t}}=\frac{g_{i l}-g_{i t}+g_{e l}-g_{e t}}{g_{i t}+g_{e t}} a_{k 1}^{2}+1=(\delta-1) a_{k 1}^{2}+1
$$

where

$$
\delta=\frac{g_{i l}+g_{e l}}{g_{i t}+g_{e t}}
$$

is the ratio of the total longitudinal conductivity to the total transverse conductivity. Similar expressions hold for the off-diagonal elements. Hence

$$
\frac{\mathbf{M}_{i}+\mathbf{M}_{e}}{g_{i t}+g_{e t}}=\left(\begin{array}{ccc}
(\delta-1) a_{11}^{2}+1 & (\delta-1) a_{11} a_{21} & (\delta-1) a_{11} a_{31} \\
(\delta-1) a_{11} a_{21} & (\delta-1) a_{21}^{2}+1 & (\delta-1) a_{21} a_{31} \\
(\delta-1) a_{11} a_{31} & (\delta-1) a_{21} a_{31} & (\delta-1) a_{31}^{2}+1
\end{array}\right)
$$

The reason for making such a normalization becomes apparent when the values of $\delta$ are considered for each set of conductivity values (Table 1). Averaging $\delta$ over the three sets gives $\delta=3.21 \pm 0.08$, which is a much tighter range than the range for the value of $\epsilon$ proposed by Roth [10]. It is interesting to note that Roberts and Scher [9] comment that this ratio, $\delta$, is 3.2 and further remark that it was the same value as in their previous work [8]. Hence, to a

good approximation, the tensor $\frac{\mathbf{M}_{i}+\mathbf{M}_{e}}{g_{i t}+g_{e t}}$ is independent of the conductivity set chosen. Further, the entire left hand side of the normalized passive bidomain equation is almost independent of the conductivity set chosen.

\subsection{A Non-dimensional Formulation}

To take into account the observations discussed in the previous section, consider the dimensionless quantities

$$
\mathbf{N}_{h}=\frac{\mathbf{M}_{h}}{g_{i t}+g_{e t}}
$$


where $h=i$ or $e$ and

$$
\Phi_{j}=\frac{\phi_{j}}{\Delta \phi_{p}}
$$

where $j=e$ or $m$ and $\Delta \phi_{p}$ is the difference in transmembrane potentials between normal and ischemic tissue during the ST segment. The passive bidomain equation (1) then becomes

$$
\nabla \cdot\left(\mathbf{N}_{e}+\mathbf{N}_{i}\right) \nabla \Phi_{e}=-\nabla \cdot \mathbf{N}_{i} \nabla \Phi_{m}
$$

where $\mathbf{N}_{i}$ has the form

$$
\mathbf{N}_{i}=\left(\begin{array}{ccc}
\frac{g_{i l}-g_{i t}}{g_{i t}+g_{e t}} a_{11}^{2}+\frac{g_{i t}}{g_{i t}+g_{e t}} & \frac{g_{i t}}{g_{i t}+g_{e t}} a_{11} a_{21} & \frac{g_{i t}}{g_{i t}+g_{e t}} a_{11} a_{31} \\
\frac{g_{i t}}{g_{i t}+g_{e t}} a_{11} a_{21} & \frac{g_{i l}-g_{i t}}{g_{i t}+g_{e t}} a_{21}^{2}+\frac{g_{i t}}{g_{i t}+g_{e t}} & \frac{g_{i t}}{g_{i t}+g_{e t}} a_{21} a_{31} \\
\frac{g_{i t}}{g_{i t}+g_{e t}} a_{11} a_{31} & \frac{g_{i t}}{g_{i t}+g_{e t}} a_{21} a_{31} & \frac{g_{i l}-g_{i t}}{g_{i t}+g_{e t}} a_{31}^{2}+\frac{g_{i t}}{g_{i t}+g_{e t}}
\end{array}\right)
$$

Also, within the blood mass

$$
\nabla^{2} \Phi_{b}=0
$$

where $\Phi_{b}=\phi_{b} / \Delta \phi_{p}$. Continuity of potential and current across the tissueblood interface require that

$$
\Phi_{e}=\Phi_{b} \text { and } \frac{g_{e t}}{g_{i t}+g_{e t}} \nabla \Phi_{e}=\frac{g_{b}}{g_{i t}+g_{e t}} \nabla \Phi_{b}
$$

where $g_{b}$ is the conductivity of blood, set to $0.62 \mathrm{mS} / \mathrm{cm}$.

As discussed in the previous section, it can be assumed that the tensor $\mathbf{N}_{e}+\mathbf{N}_{i}$ is independent of the choice of conductivity data set, whereas the right hand side tensor cannot. Hence the effect the different sets of conductivity data have on the extracellular potential distribution can be studied by visualising the right hand side of equation (14).

In general, with the aid of the finite volume method $[14,15]$, the right hand side of equation (14) can be evaluated numerically for a specified geometry using the stiffness matrix for the particular conductivity set and then 
multiplying by the vector representing the transmembrane potential distribution. However, for simple geometries, the right hand side of equation (14) can be evaluated analytically.

\section{Results}

As a simple example consider a slab of cardiac tissue of size $16 \mathrm{~cm} \times 16 \mathrm{~cm}$ in the $x$ and $y$ directions and of thickness $1 \mathrm{~cm}$ as described previously [3]. The governing equations are solved via the finite volume method which has been previously validated [15]. The blood mass has a depth of $26 \mathrm{~cm}$ and at the bottom face of the blood mass the potential was set to zero. The ischemic region was located centrally within the cardiac tissue slab having a size of $4 \mathrm{~cm} \times 4 \mathrm{~cm}$ and covering $50 \%$ of the depth of the tissue from the endocardium. The ischemic boarders were given by $\lambda_{x}=\lambda_{y}=\lambda_{z}=0.01$. For this example fiber rotation was ignored.

Figure 1 shows dimensionless epicardial potential distributions, obtained by solving (14), for the three experimental data sets from Clerc [7] (panel (a)), Roberts et al. [8] (panel (b)) and Roberts and Scher [9] (panel (c)). It can be seen from this figure that the data of Clerc (panel (a)) and of Roberts et al. (panel (b)) give epicardial distributions which are topologically very similar in that there are three peaks in the potential distribution and two small wells of negative potential. (Note that the problem has been normalized with respect to the transmembrane potential difference of $-30 \mathrm{mV}$, so here positive values represent ST depression). The data of Roberts and Scher (panel (c)) give rise to a topologically different potential distribution with one peak in the potential distribution and no potential wells. 


\section{Discussion}

In order to make some sense of the results presented in the previous section, consider the right hand side of equation (14) for this simplified model:

$$
-\nabla \cdot \mathbf{N}_{i} \nabla \Phi_{m}=-G_{i l} \frac{\partial^{2} \Phi_{m}}{\partial x^{2}}-G_{i t} \frac{\partial^{2} \Phi_{m}}{\partial y^{2}}-G_{i t} \frac{\partial^{2} \Phi_{m}}{\partial z^{2}}
$$

where $G_{i q}=\frac{g_{i q}}{g_{i t}+g_{e t}}($ for $q=l$ or $t)$ and $\Phi_{m}=\Psi(x) \Psi(y) \Psi(1-z)$. Here, $\Psi(t)$ is a sigmoidal function representing the change in electrical properties from normal to ischemic tissue and having the property that $\Psi(0)=1[3]$. Now, consider the value of $\nabla \cdot \mathbf{N}_{i} \nabla \Phi_{m}$ along three key lines in the simplified geometry:

1. Along the line $y=0, z=1$ :

$$
-\nabla \cdot \mathbf{N}_{i} \nabla \Phi_{m}=-G_{i l} \frac{\partial^{2} \Psi}{\partial x^{2}}
$$

2. Along the line $x=0, z=1$ :

$$
-\nabla \cdot \mathbf{N}_{i} \nabla \Phi_{m}=-G_{i t} \frac{\partial^{2} \Psi}{\partial y^{2}}
$$

3. Along the line $x=0, y=0$ :

$$
-\nabla \cdot \mathbf{N}_{i} \nabla \Phi_{m}=-G_{i t} \frac{\partial^{2} \Psi}{\partial z^{2}}
$$

where the fact that $\Psi(0)=1$ has been used. The value of the right hand side of equation (14) along each of the above lines in the neighbourhood of the ischemic boundary is plotted in Figure 2 for each set of experimental data. The figure shows that the plot of equation (18) through the $y$ face, line 2, (panel (b)) has the same magnitude, but opposite direction, as the plot through the $z$ face, line 3 , (panel $(\mathrm{c})$ ). This is not surprising since both quantities are proportional to $G_{i t}$. However, the plot of equation (18) through the $x$ face, line 1 , has a different magnitude as it is related to $G_{i l}$. 
According to Hopenfled et al. $[1,2]$, the shape of the epicardial potential distribution depends on the ratio of the current flow across the lateral and transmural boundaries between the normal and ischemic regions of the tissue. For the example under consideration this ratio is given by

$$
\frac{G_{i l} \frac{\partial^{2} \Phi_{m}}{\partial x^{2}}}{G_{i t} \frac{\partial^{2} \Phi_{m}}{\partial z^{2}}}=\frac{g_{i l}}{g_{i t}}
$$

Values of the ratio $\frac{g_{i l}}{g_{i t}}$ are given in Table 1 . From this it can be seen that the ratio for the data of Clerc (9.0) is similar to the ratio for the data of Roberts et al. (10.8). Both of these values are markedly different from the ratio obtained from the data of Roberts and Scher (5.7). Hence, for this simple example, the difference in epicardial potential distributions can be explained by the differences in the ratios of $g_{i l}$ to $g_{i t}$ for the different data sets.

Interestingly, the ratio $\frac{g_{i l}}{g_{i t}}$ occurs quite naturally in this non-dimensional formulation of the passive bidomain equation, as does the ratio $\frac{g_{e t}}{g_{i t}}$. The dimensionless groups which arise in the tensor $\mathbf{N}_{i}$ are

$$
\frac{g_{i l}-g_{i t}}{g_{i t}+g_{e t}}=\frac{\frac{g_{i l}}{g_{i t}}-1}{1+\frac{g_{e t}}{g_{i t}}}
$$

and

$$
\frac{g_{i t}}{g_{i t}+g_{e t}}=\frac{1}{1+\frac{g_{e t}}{g_{i t}}}
$$

Values for the ratio $\frac{g_{e t}}{g_{i t}}$ are also given in Table 1 . However, this ratio does not appear to influence the topology of the epicardial potential distributions in any obvious manner.

It is interesting to note that the ratio $\delta$ also appears in the work of Potse et al. [13], where it is labelled $R^{\prime}$ and called the "myocardial bulk conductivity". The value quoted for $R^{\prime}$ was 4 , based on the conductivity values suggested by Roth [10], which is a direct consequence of choosing a value for $\epsilon$ of 0.75 . 


\section{Conclusions}

This paper has presented a new non-dimensional formulation of the passive bidomain equation for modeling the electric field due to subendocardial ischemia in cardiac tissue. The new formulation divides the governing equation (equation (1)) by the quantity $g_{i t}+g_{e t}$ which has the effect of rendering the left hand side of the equation approximately independent of the choice of the measured cardiac conductivity values. From this it follows that any effects different conductivity sets have on the epicardial potential distribution can be investigated by studying the right hand side of the passive bidomain equation.

Recent studies [16] have considered determining two further conductivity values in the direction normal to the sheets of cardiac fibers. These values are believed to be different to the usual transverse conductivity values due to the presence of cleavage planes. However, the values of the longitudinal and transverse conductivities used in these studies are based on the ideas of Roth [10]. Inclusion of these new conductivity values will provide an interesting extension to the work presented here.

As a final conclusion, consider a new set of nominal conductivity values. Following Roth [10], set $g_{i l}=1.0, g_{i t}=0.1$ and $g_{e l}=1.0$. The remaining value comes from the consistent experimental observation that

$$
\delta=\frac{g_{i l}+g_{e t}}{g_{i t}+g_{e t}}=3.2
$$

which in turn gives $g_{e t}=0.525$.

\section{References}

[1] B. Hopenfeld, J. G. Stinstra, R. S. MacLeod, The effect of conductivity on st-segment epicardial potentials arising from subendocardial 
ischemia, Annals of Biomedical Engineering 33 (6) (2005) 751-763.

[2] B. Hopenfeld, J. G. Stinstra, R. S. Mac $\{$ L $\}$ eod, Mechanism for $\{\mathrm{ST}\}$ depression associated with contiguous subendocardial ischemia, Journal of Cardiovascular Electrophysiology 15 (2004) 1200-1206.

[3] P. R. Johnston, D. Kilpatrick, C. Y. Li, The importance of anisotropy in modeling ST segment shift in subendocardial ischemia, IEEE Transactions on Biomedical Engineering 48 (12) (2001) 1366-1376.

[4] P. R. Johnston, D. Kilpatrick, The effect of conductivity values on ST segment shift in subendocardial ischemia, IEEE Transactions on Biomedical Engineering 50 (2) (2003) 150-158.

[5] P. R. Johnston, A cylindrical model for studying subendocardial ischemia in the left ventricle, Mathematical Biosciences 186 (1) (2003) $43-61$.

[6] M. Potse, R. Coronel, S. Falcao, A. R. LeBlanc, A. Vinet, The effect of lesion size and tissue remodeling on ST deviation in partial-thickness ischemia; Heart Rhythm 4 (2) (2007) 200-206.

[7] L. Clerc, Directional differences of impulse spread in trabecular muscle from mammalian heart, Journal of Physiology 255 (1976) 335-346.

[8] D. E. Roberts, L. T. Hersh, A. M. Scher, Influence of cardiac fiber orientation on wavefront voltage, conduction velocity and tissue resistivity in the dog, Circ. Res. 44 (1979) 701-712.

[9] D. E. Roberts, A. M. Scher, Effects of tissue anisotropy on extracellular potential fields in canine myocardium in situ, Circ. Res. 50 (1982) 342351. 
[10] B. J. Roth, Electrical conductivity values used with the bidomain model of cardiac tissue, IEEE Transactions on Biomedical Engineering 44 (4) (1997) 326-328.

[11] R. M. Gulrajani, Bioelectricity and Biomagnetism, John Wiley and Sons, 1998.

[12] P. Colli-Franzone, L. Guerri, Spreading of excitation in 3-D models of the anisotropic cardiac tissue I: Validation of the eikonal model, Mathematical Biosciences 113 (1993) 145-209.

[13] M. Potse, B. Dubé, A. Vinet, Cardiac anisotropy in boundary-element models for the electrocardiogram, Medical \& Biological Engineering \& Computing 47 (7) (2009) 719-729.

[14] R. C. Penland, D. M. Harrild, C. S. Henriquez, Modeling impulse propagation and extracellular potential distributions in anisotropic cardiac tissue using a finite volume element discretization, Computing and Visualization in Science 4 (4) (2002) 215-226.

[15] P. R. Johnston, A finite volume method solution for the bidomain equations and their application to modeling cardiac ischemia, Computer Methods in Biomechanics and Biomedical Engineering 13 (2) (2010) $157-170$.

[16] D. A. Hooks, K. A. Tomlinson, S. G. Marsden, I. J. LeGrice, B. H. Smaill, A. J. Pullan, P. J. Hunter, Cardiac microstructure: Implications for electrical propagation and defibrillation in the heart, Circulation Research 91 (4) (2002) 331-338. 


\section{Table and Figure Captions}

Table 1: Conductivity data from three experimental studies (given in $\mathrm{mS} / \mathrm{cm}$ ) and derived quantities used in this study.

Figure 1: Dimensionless epicardial potential distributions for a slab of cardiac tissue obtained by solving the non-dimensional passive bidomain equation (14) for various sets of conductivity data as indicated. Positive potentials are shown with solid lines and the thick solid line represents the zero of potential.

Figure 2: Plots of the right hand side of the non-dimensional passive bidomain equation (14) along the indicated lines in a slab model of cardiac tissue. 


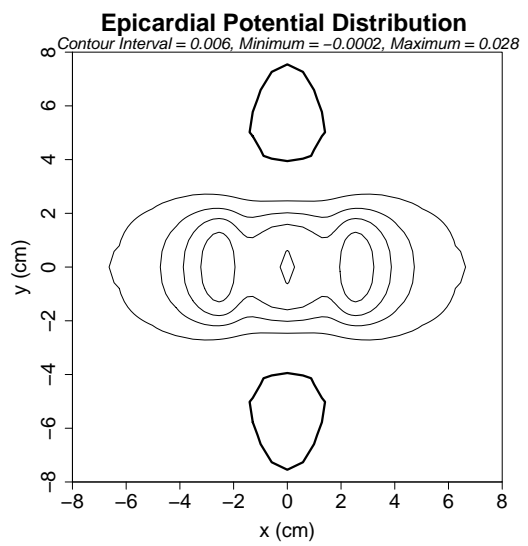

(a) Clerc (1976) [7]

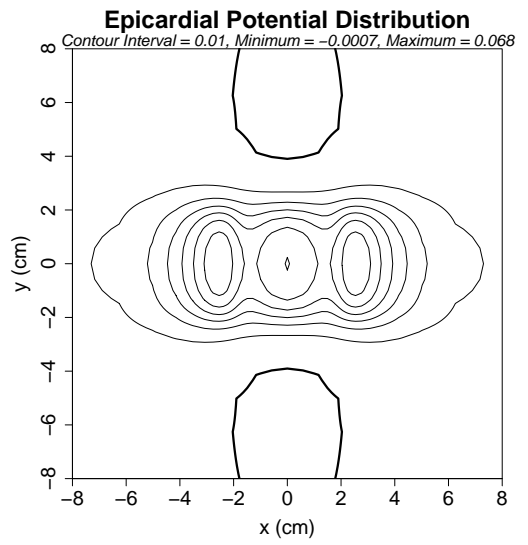

(b) Roberts et al. (1979) [8]

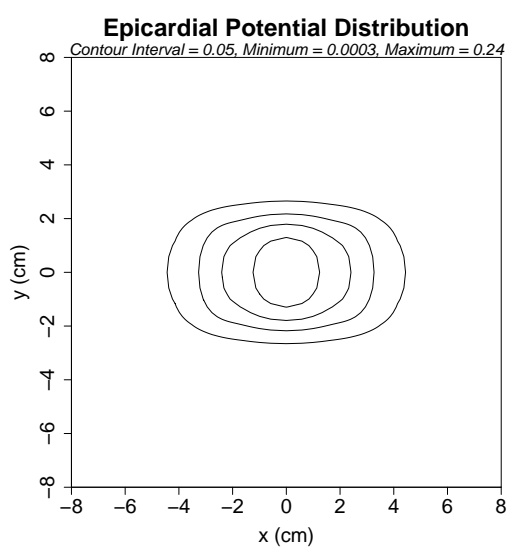

(c) Roberts \& Scher (1982) [9]

Figure 1: 


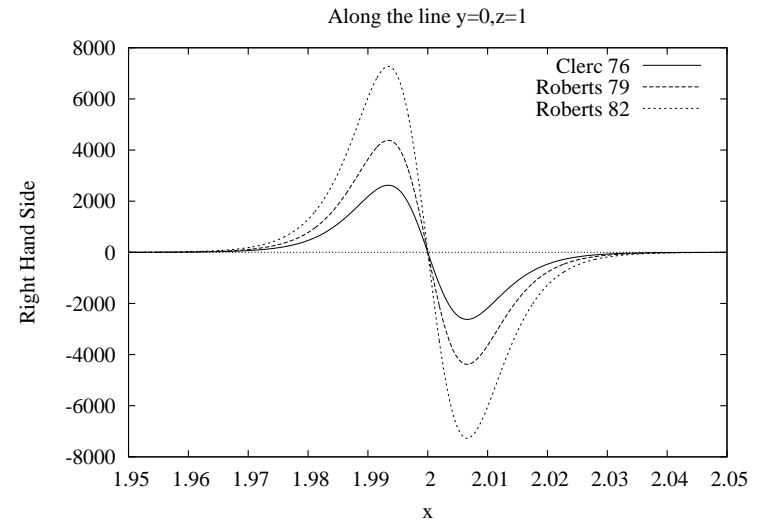

(a) Plot through $x$ face, along the line $y=0, z=1$

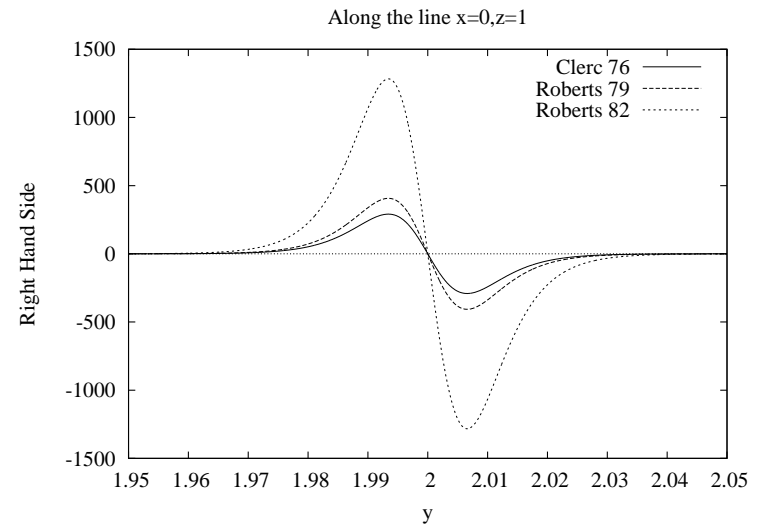

(b) Plot through $y$ face, along the line $x=0, z=1$

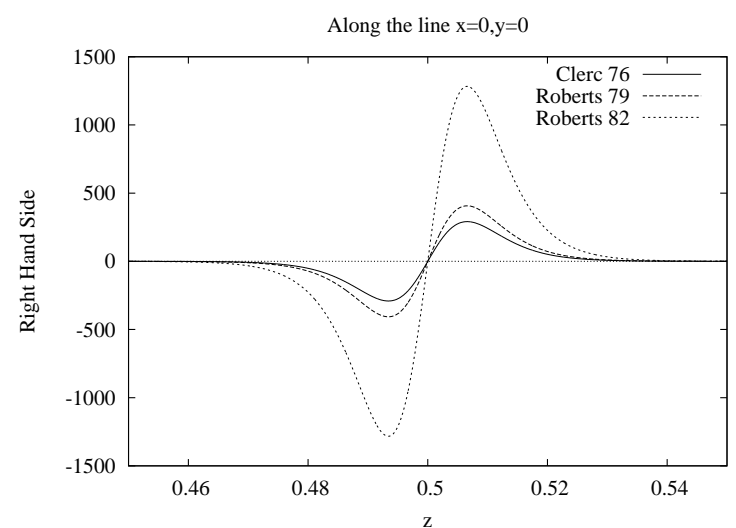

(c) Plot through $z$ face, along the line $x=0, y=0$

Figure 2: 


\begin{tabular}{|c|c|c|c|}
\hline Quantity & $\begin{array}{c}\text { Clerc } \\
(1976)\end{array}$ & $\begin{array}{c}\text { Roberts et al. } \\
(1979)\end{array}$ & $\begin{array}{c}\text { Roberts \& Scher } \\
(1982)\end{array}$ \\
\hline$g_{i l}$ & 0.17 & 0.28 & 0.34 \\
$g_{i t}$ & 0.019 & 0.026 & 0.06 \\
$g_{e l}$ & 0.62 & 0.22 & 0.12 \\
$g_{e t}$ & 0.24 & 0.13 & 0.08 \\
\hline$\epsilon$ & 0.71 & 0.84 & 0.74 \\
\hline$\delta$ & 3.13 & 3.21 & 3.29 \\
\hline$g_{i l} / g_{i t}$ & 9.0 & 10.8 & 5.7 \\
$g_{e t} / g_{i t}$ & 12.3 & 5.0 & 1.3 \\
\hline
\end{tabular}

Table 1: 